\title{
Propagation de pollution dans un aquifère alluvial. L'effet de parcours
}

\author{
The propagation of pollution in an alluvial aquifer. \\ Distance effect
}

\author{
A. Dieulin \\ Laboratoire d'hydrologie mathématique \\ Ecole nationale supérieure des mines de Paris*
}

Le Prix Henri Milon de la Société hydrotechnique de France a été décerné à Monsieur A. Dieulin pour son mémoire de thèse: Propagation de pollution dans un aquifère alluvial. L'effet de parcours. La Houille Blanche félicite chaleureusement $M$. Dieulin pour cette distinction et le remercie d'avoir bien voulu rédiger - à l'attention de ses lecteurs - un large résumé de son mémoire.

Cette étude [1] résume une thèse de DocteurIngénieur [2] préparée sous la direction de MM. Beaudoin et de Marsily, professeurs à l'Ecole des Mines de Paris, et soutenue devant l'Ecole des Mines et l'Université de Paris VI, à Paris, le 30 juin 1980.

L'étude exposée porte sur le mouvement de solutions dans des milieux poreux saturés naturels. Le transfert de masse en milieu poreux a fait l'objet d'une théorie maintenant classique [3]; elle décrit le phénomène à l'aide d'une équation dite "équation de la dispersion". Les propriétés dispersives du milieu poreux considéré y sont représentées par des coefficients dits "de dispersion". Leurs valeurs ont été mesurées sur le terrain et en laboratoire : celles déterminées directement sur le terrain se sont avérées être de plusieurs ordres de grandeurs différentes de celles tirées en laboratoire d'une carotte du même terrain. De plus, les coefficients de dispersion, qui résultent de l'interprétation d'essais de traçage sur le terrain, semblent augmenter avec la distance entre les points d'injection et d'observation ; cette croissance, depuis longtemps constatée, est un des obstacles majeurs à la possibilité d'une prévision fiable de la propagation d'une pollution dans des cas réels. On considère habituellement que cette croissance correspond à un changement

\footnotetext{
* 35, Rue St.Honoré, 77305 Fontainebleau.
}

dans l'échelle des hétérogénéités prises en compte au cours de la propagation de la pollution : c'est le fameux "effet d'échelle".

Cette vision chaotique du milieu poreux ne correspond pas à l'observation que l'on peut faire notamment du milieu alluvial : ce dernier est formé par l'assemblage de corps sédimentaires de grande extension (paléochenaux), et ce n'est pas en terme d'hétérogénéités, mais de structures, qu'il convient de le décrire. Aussi l'interprétation de la croissance du coefficient de dispersion sous-tendue par le terme "d'effet d'échelle" ne nous a pas semblé satisfaisante : ce problème devait être replacé dans son contexte géologique.

C'est ainsi que, sur un site alluvial, nous avons d'une part reconnu l'organisation du sédiment et procédé, d'autre part, à un traçage par injection de sel. Les enregistrements effectués in situ ont conduit à des résultats expérimentaux dont l'interprétation ne pouvait se suffire des formulations habituelles.

Cette étude présentera donc successivement les résultats qualitatifs et quantitatifs de notre essai de traçage, la mise en évidence et la généralisation de la loi temporelle de dispersion correspondant à la notion nouvelle d'effet de parcours.

\section{Interprétation en termes structuraux}

Nous avons réalisé, sur le site de Torcy, un traçage dans les alluvions de la Marne pendant un mois et demi environ [4]. L'aquifère est une nappe libre de $5 \mathrm{~m}$ d'épaisseur, à écoulement à peu près uniforme (gradient $1,3 \%$ ), étudiée sur un dispositif de 13 forages (Fig. 1). La perméabilité moyenne y est d'environ $0,7.10^{-3} \mathrm{~m} / \mathrm{s}$. L'expérience rapportée consiste en l'injection instantanée de $2 \mathrm{~m}^{3}$ de saumure à $20 \mathrm{~g} / 1$ dans le forage 1 


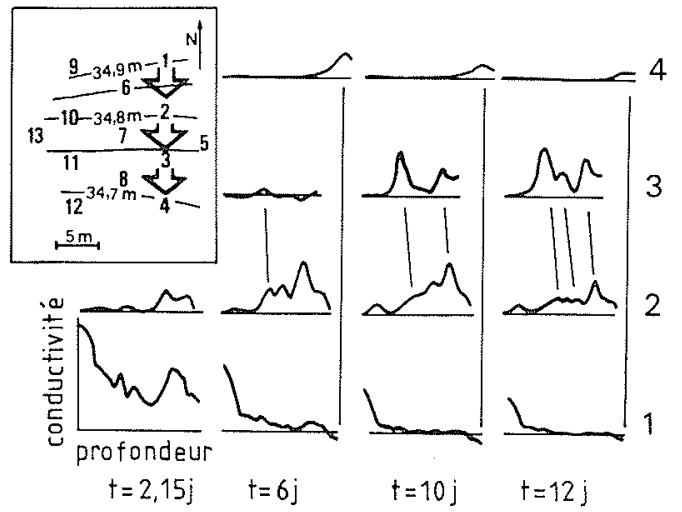

Figure 1 - Evolution du nuage de saumure aux différents forages.

Cartouche: le site expérimental et la piézométrie.

(Fig. 1). La saumure, suivie in situ par diagraphies, n'a été détectée que dans les forages 2, 3 et 4 .

Les logs de conductivité apparente au forage d'injection montrent un retour rapide à l'état initial (Fig. 1). Aux deux forages suivants, des arrivées de sel sont détectées à différentes cotes : leur position stable au cours du temps montre l'existence de guides au sein du sédiment, que l'on peut suivre entre ces deux points.

Les logs bruts peuvent être décomposés, sans trop d'ambiguité, en contributions élémentaires (Fig. 2A). Les variations de la conductivité apparente en fonction du temps, tracées pour chacune d'elles, caractérisent leurs propriétés hydrodispersives : la similitude de certaines courbes (Fig. 2B) permet alors le rapprochement des guides correspondants (guides II). De même, le retour à l'état initial du forage d'injection n'est pas uniforme : des niveaux plus conducteurs correspondant sans doute à un départ préférentiel de saumure montre une disposition analogue à celle des guides déjà rencontrés. La corrélation spatiale des niveaux ainsi soulignés aux divers forages (Fig. 3) confirme alors la continuité de guides stratifiés.

Les logs de rayonnement $\gamma$ naturel de la formation (lié à sa teneur en argile) permettent de décomposer le sédiment en séquences : tranche de sédiment comprise entre deux discontinuités majeures et composée, quand elle est complète, d'un terme basal grossier, d'un second terme sableux et d'un dernier terme argileux. La superposition du cheminement du nuage de

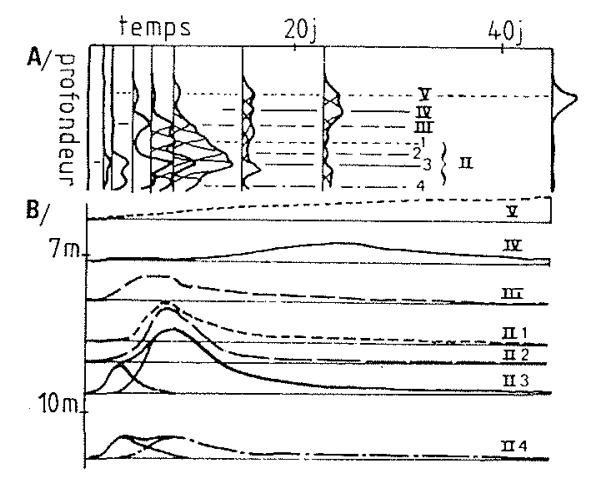

Figure 2 - Arrivées de saumure au forage 2.

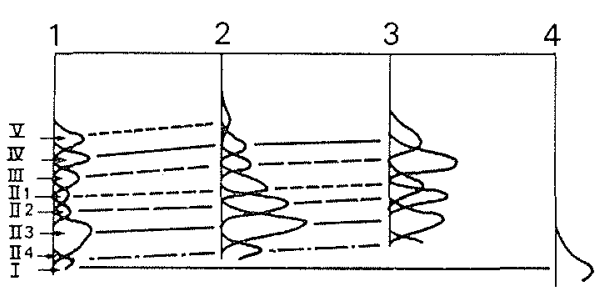

Figure 3 - Propagation du nuage de saumure dans le plan des forages $1,2,3$ et 4 .

saumure au schéma sédimentaire séquentiel ainsi obtenu montre clairement l'identité des guides de pollution et de la base perméable des corps sédimentaires.

Les courbes de variation dans le temps de la conductivité de l'eau pompée aux forages 2,3 et 4 , dites "courbes de restitution", peuvent être décomposées en deux contributions principales. La plus rapide est en relation avec le guide basal (I) ; l'autre pic correspond, aux forages 2 et 3 , à la saumure propagée dans les guides II et, au forage 4 , à une partie de cette saumure descendue dans le guide basal (Fig. 1).

La mise en évidence de guides au sein du milieu allu. vionnaire et de leur quasi-indépendance rend donc possible et légitime la quantification séparée de la dispersion du traceur dans chacun d'eux. Etant donné leur épaisseur réduite et leur caractère quasiment plan, le traçage dans ces corps a été modélisé par une injection brève de traceur, dans un milieu plan homogène où la vitesse d'écoulement est prise constante.

\section{Interprétation hydrodispersive classique}

Le calage sur des abaques [5] des courbes de variation de la conductivité apparente au cours du temps, en un point, fournit alors les paramètres équivalents de la portion de guide comprise entre le point d'injection et le point d'observation : $u$ (vitesse d'écoulement), $\alpha_{L}$ (dispersivité longitudinale) et

$$
F=\frac{M / \omega}{\sqrt{\alpha_{T}}}
$$

(caractérisant l'amplitude du signal : $M$ est la masse injectée par unité d'épaisseur, $\omega$ la porosité, $\alpha_{T}$ la dispersivité transversale). Les valeurs obtenues pour divers guides, sur différentes portions du site, montrent une croissance de la dispersivité équivalente longitudinale avec la distance (tableau 1) : "l'effet d'échelle" est directement observable au sein d'un même corps sédimentaire. Par ailleurs, l'exploitation des courbes de "restitution" peut se faire en admettant que la concentration mesurée est due à la superposition d'arrivées indépendantes, et donne alors des valeurs de paramètres équivalents très voisines de celles déterminées directement dans les guides.

La structure sédimentaire, dont l'influence sur le cheminement du nuage de dispersion est tout à fait fondamentale, fournit le cadre dans lequel doit s'inscrire l'étude physique de la dispersion. "L'effet d'échelle" est observable au sein même de chaque corps sédimentaire : on ne peut plus se limiter à attribuer à 


\begin{tabular}{|c|r|r|r|r|r|r|r|r|r|r|r|}
\hline \multicolumn{2}{|c|}{ Tableau I. - Valeurs de la dispersivité longitudinale équivalente, de la vitesse movenne de pores équivalente et du facteur } \\
de norme équivalent pour les différents tronçons de guide.
\end{tabular}

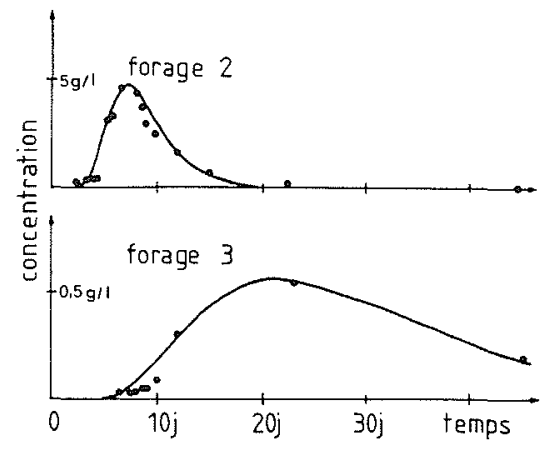

Figure 4 - Ajustement de solutions classiques du modèle homogène sur les données expérimentales dans le guide II.1.

un milieu réel ("statistiquement homogène") un coefficient de dispersion unique.

Celui-ci varie pour le moins avec la distance sans qu'il soit nécessaire d'imaginer d'éventuels changements d'échelle. Cependant si les solutions du modèle homogène établies avec les paramètres équivalents obtenus paraissent s'ajuster aux courbes expérimentales (Fig. 4), les écarts systématiques observés conduisent à s'interroger sur la constance dans le temps de ces paramètres en un point donné [6].

\section{Interprétation hydrodispersive partielle}

La montée de la courbe expérimentale (Fig. 4) est plus tardive et plus brutale, et sa descente est plus tardive et plus douce. On peut alors caler une solution du modèle homogène sur une partie seulement des points expérimentaux (Fig. 5-1 et 2): c'est une solution analytique partielle, et les paramètres équivalents partiels déterminés sont attribués au point moyen de la portion de la courbe calée. Ainsi, pour un guide donné, la dispersivité équivalente longitudinale partielle $\alpha_{L}$ sera fonction de la distance et aussi du temps.

Les courbes expérimentales obtenues dans le guide II.1 permettent de déterminer, en chaque forage, les variations de $\alpha_{L}$ au cours du temps (Fig. 5-3). Les portions de courbes obtenues aux forages 2 et 3 s'engrènent parfaitement : il apparaît donc, qu'en première approximation, la dispersivité partielle $\alpha_{L}$ ne dépend que du temps. On peut alors donner une explication de l'apparente dépendance du coefficient de dispersion global avec la distance, $x$, au point d'obser-

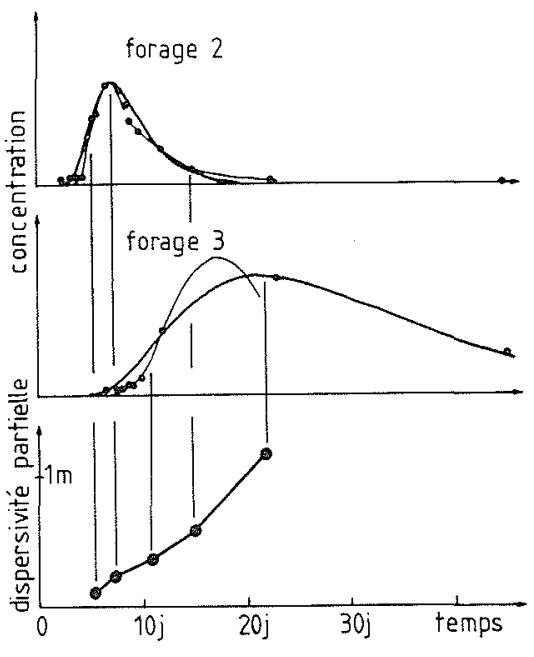

Figure 5 - Analyse "partielle" du guide II.1.

vation. Sa valeur correspond à un échantillonnage unique de la loi de variation temporelle de $\alpha_{L}$ approximativement au temps $t=x / u$, où $u$ est la vitesse moyenne de pores équivalente. Les différentes valeurs du paramètre global étant déterminées pour des distances $x$ croissantes, elles correspondent donc à des temps de plus en plus grands : la loi de variation temporelle de la dispersivité partielle étant généralement croissante, il s'ensuit donc une augmentation du paramètre global que l'on relie brutalement à celle de $x$.

Une analyse identique peut être menée sur l'ensemble des guides. On détermine pour chacun d'eux une loi de variation temporelle de $\alpha_{L}$ (Fig. 6). Les

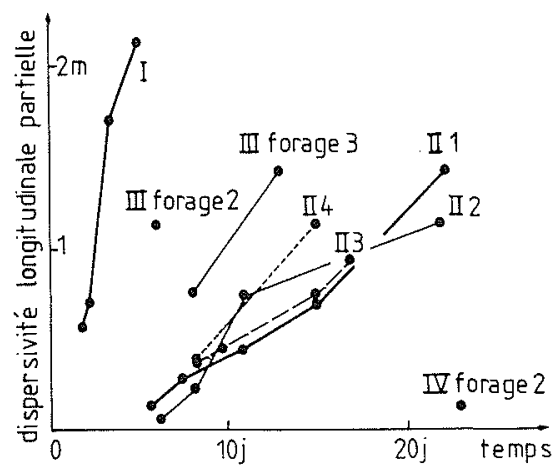

Figure 6 - Analyse "partielle" des guides. 
diverses courbes se distribuent alors dans l'ordre des vitesses au sein des guides : le guide (l) étant le plus rapide et le guide (IV) le plus lent. On note qu'en aucun cas sur la distance tracée $(15 \mathrm{~m})$, il n'apparait de "palier" dans la croissance de $\alpha_{L}$.

\section{La loi temporelle de dispersion}

La démarche précédente montre la variation cohérente d'un paramètre équivalent partiel dans les divers guides. La résolution de l'équation classique de la dispersion, pour un milieu plan homogène à vitesse réelle $u$ fixe parallèle à l'axe $x$, et pour une injection instantanée de traceur conduit à une répartition spatiale du nuage qui suit une loi de Gauss à 2 dimensions : les variances longitudinale et transversale du nuage étant respectivement :

où

$$
\sigma_{X}^{2}=2 D_{L} t \quad \text { et } \quad \sigma_{Y}^{2}=2 D_{T} t,
$$

$$
D_{L}=\alpha_{L} u \quad \text { et } \quad D_{T}=\alpha_{T} u
$$

sont les coefficients de dispersion longitudinal et transversal.

L'analyse par calages partiels précédente suggère en fait que la variance longitudinale du nuage de traceur est plus que linéairement proportionnelle au temps.

Nous avons donc été amenés à proposer la représentation de la distribution à l'instant $t$ d'un traceur injecté ponctuellement et de manière brève (à $t=0$ ) dans un milieu plan, siège d'un écoulement à vitesse $u$ constante, par une loi de Gauss à 2 dimensions, dont :

(i) le point moyen se déplace à la vitesse $u$;

(ii) la variance longitudinale est de la forme :

$$
\sigma_{L}^{2}=2 D_{L}(t) \cdot t
$$

où $D_{L}(t)$ est une fonction du temps que l'on désignera comme la loi temporelle de dispersion;

(iii) la variance transversale est proportionnelle au temps :

$$
\sigma_{T}^{2}=2 D_{T} \cdot t
$$

où $D_{T}$ est une constante

Sous les hypothèses précédentes, la répartition du traceur le long de l'axe d'écoulement issu du point d'injection s'écrit :

$$
\begin{aligned}
& C(x, t)=g_{\left[D_{L}(t), x\right]}(t)= \\
& \quad \frac{M / \omega}{4 \pi \sqrt{D_{T}}} \frac{1}{t \sqrt{D_{L}}(t)} e^{-(x-u t)^{2} / 4 t D_{L}(t)}
\end{aligned}
$$

où $M$ est la masse injectée par unité d'épaisseur, et $\omega$ la porosité.

Dans le cas d'une loi $D_{L}(t)$, fonction simple du temps, les courbes $g_{\left[D_{L}(t), x\right]}(t)$ ont été tracées pour deux distances d'observation (Fig. 7). Les courbes $g_{\left[D_{L}\left(t_{0}\right), x\right]}(t)$, tracées pour une valeur constante $D_{L}\left(t_{0}\right)$ correspondent aux courbes partielles de l'analyse précédente. Des abaques ont été établies pour permettre une détermination aisée de la loi temporelle $D_{L}(t)$ (Fig. 8). Les données relatives au guide II.1, aux forages 2 et 7 , ont permis d'évaluer la loi temporelle de dispersion du guide, et les courbes cal-

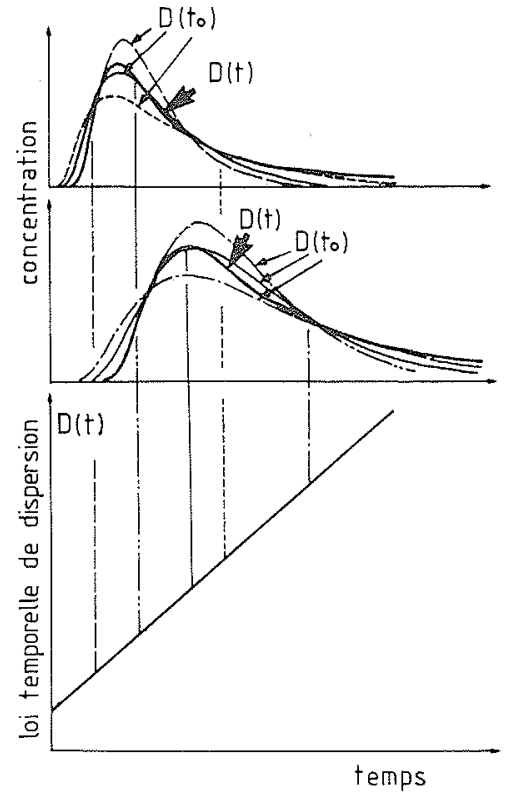

Figure 7 - Réponses calculées à 2 distances d'observation à l'aide d'une fonction de dispersion simple $(D(t))$.

Comparaison avec les solutions classiques du modèle homogène $\left(D\left(t_{0}\right)\right)$.

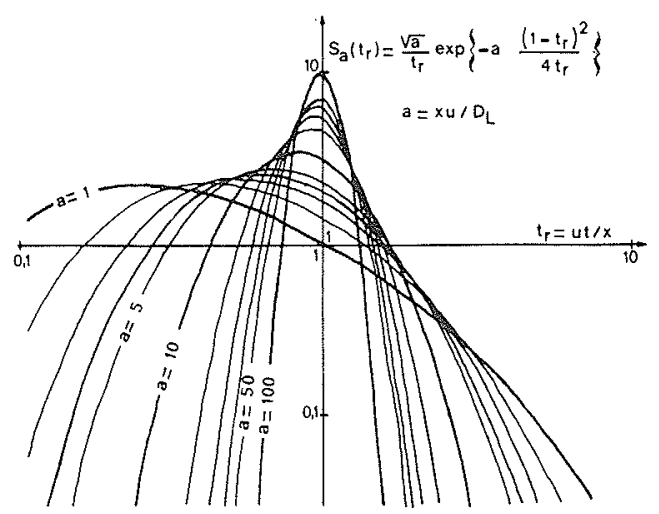

Figure 8 - Abaque bilogarithmique pour la détermination pratique de la loi temporelle de dispersion.

culées pour ces deux distances leur ont été superposées (Fig. 9). Les courbes calculées s'ajustent parfaitement aux points expérimentaux.

\section{L'effet de parcours}

Cette formulation rejoint des études théoriques récentes ([7], [8]) qui montrent, pour un milieu poreux fortement hétérogène, que le mouvement d'un traceur ne suit pas l'équation classique de la dispersion et que les propriétés dispersives du milieu peuvent être caractérisées par des fonctions du temps. On doit alors s'interroger sur la signification physique de cette dépendance temporelle. Elle résume en fait l'histoire du nuage de pollution depuis le moment de l'injection, et l'on peut lui substituer une dépendance en distance moyenne parcourue par le nuage de traceur. En effet, le nuage de traceur dans un guide a une posi- 


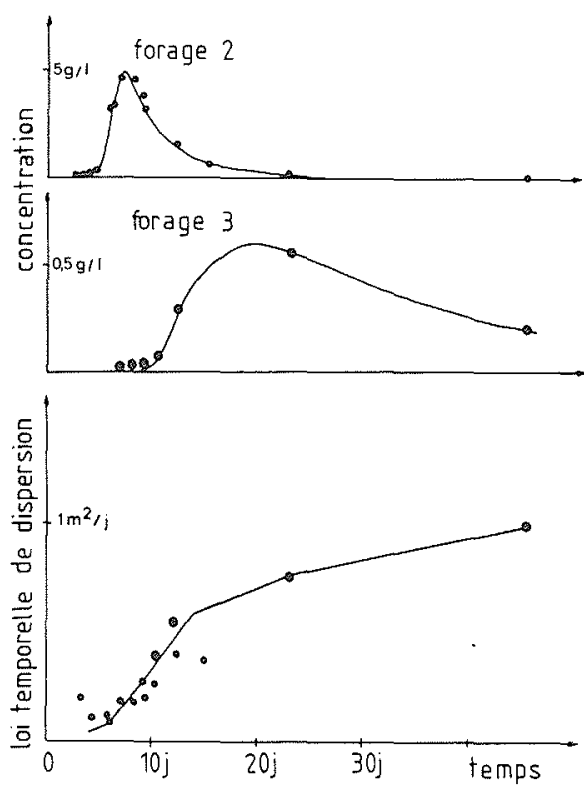

Figure 9 - Loi temporelle de dispersion déterminéa pour le guide II.1.

Réponses calculées aux forages 2 et 3 . Comparaison aux points mesurés.

tion moyenne définie par $\bar{x}=u t$, et la loi de dispersion, $D_{L}$, est fonction de $t$, c'est-à-dire de $\bar{x} / u$. Ainsi, cette variation temporelle, qui pourrait apparaitre en opposition avec la variation spatiale (l'effet de changement d'échelle) du coefficient de dispersion, correspond, comme dans ce dernier cas, à une prise en compte croissante des hétérogénéités du milieu, que ce soit par le changement de corps sédimentaires, ou en raison de la variabilité interne du guide. L'évolution observée dans ces deux cas résulterait des diverses irrégularités rencontrées au cours du transfert : c'est ce que résume la notion "d'effet de parcours".

\section{Les formes in trinsèques de dispersion}

Il devient ainsi possible, au moins dans le cas d'une injection brève, de proposer une formulation pratique permettant de prévoir le comportement du nuage de dispersion dans un guide. Des expériences anciennes de traçage à l'lode 131, effectuées en 1975 par le BRGM et le CENG sur le site de Bonnaud (Jura), ont pu être interprétées à l'aide d'une loi temporelle de dispersion. Enfin, la formulation, introduite ici dans le cadre de guides bidimensionnels, a pu être étendue au cas monodimensionnel, et a permis de rendre compte de traçages réalisés en laboratoire sur une carotte métrique de grès [9]. Ces essais in vitro, menés avec divers régimes d'écoulement, nous ont amenés à une représentation des lois temporelles de dispersion qui pourrait constituer une forme intrinsèque de dispersion. Cette représentation est évidemment relative au grès considéré, mais la démarche utilisée est facilement transposable à un autre milieu. Dans les essais in situ que nous avons menés, il n'a pas été possible de modifier le régime d'écoulement. On a pu, cependant, proposer des formes plus intrinsèques pour les lois temporelles de disper-

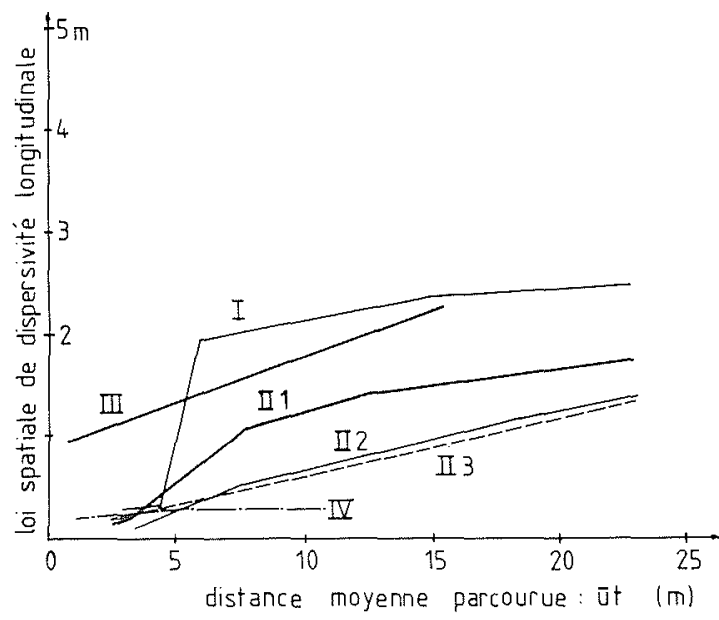

Figure 10 - Lois spatiales de dispersivité des guides.

sion en considérant le rapport de ces dernières par la vitesse de leur guide respectif en fonction de la distance moyenne parcourue par le nuage de traceur dans chaque guide (Fig. 10). Ces formes intrinsèques de dispersion présentent un intérêt pratique tout à fait important puisqu'elles pourraient permettre d'étendre l'utilisation des lois temporelles de dispersion établies sur un traçage particulier à d'autres régimes d'écoulement.

\section{Conclusion}

Pour un milieu alluvial, l'analyse du sédiment en terme de séquences permet de mettre en évidence les guides à l'intérieur desquels cheminent les éléments en solution. A échelle métrique, ces guides sont pratiquement indépendants, mais sur des distances plus grandes, c'est l'organisation des différents corps sédimentaires (ravinement, téléscopage) qui conditionne la migration.

$\mathrm{Au}$ sein d'un même guide, l'équation classique de la dispersion ne rend pas aisément compte des propagations observées. Le classique effet d'échelle, qui souligne cette divergence, interprète la croissance du coefficient de dispersion observé comme liée à la distance entre les forages d'injection et d'observation. Une analyse plus fine montre que, pour une injection brève de traceur, il s'agit en fait d'une variation temporelle qui peut etre traduite par une loi temporelle de dispersion : la répartition spatiale du traceur dans un guide d'épaisseur réduite peut alors être modélisée par une loi de Gauss à deux dimensions dont la variance longitudinale est plus que linéairement proportionnelle au temps. Cet effet de temps peut cependant être interprété comme un effet de parcours : la loi temporelle de dispersion dépendrait en fait de la distance de déplacement moyen du nuage de pollution traduisant ainsi une prise en compte croissante d'une même variabilité au sein d'un guide.

Ce nouvel outil d'analyse des propriétés dispersives du milieu poreux a vu sa généralité confirmée dans des cas expérimentaux très différents. On peut 
alors espérer la mise en évidence de telles grandeurs dans le cadre de domaines d'expérimentation plus larges, semblables à ceux concernés par des cas réels de pollution. Si cela se vérifie, la prévision de la propagation des pollutions aura grandement progressé.

\section{Références}

[1] Ce travail a été effectué grâce à une aide du "Comité Eau" du Ministère de la Culture et de l'Environnement.

[2] DIEULIN A. - Thèse de Docteur-Ingénieur : Propagation de pollution dans un aquifère alluvial : l'effet de parcours. ENSMP-Université Paris VI.

[3] FRIED J.J. - Thèse de Docteur es Sciences : Etudes théoriques et méthodologiques de la dispersion en milieu poreux naturel. Université de Bordeaux, 1972.
[4] DIEULIN A., BEAUDOIN B., De MARSILY G. - Sur le transfert d'éléments en solution dans un aquifère aliuvionnaire structuré. CR Acad. Sc.., 291, série D, p. 805, 1980.

[5] SAUTY J.P. - Thèse de Docteur-Ingénieur : Contribution l'identification des paramètres de dispersion dans les aquifères par interprétation d'expériences de traçage. Université de Grenoble, 1977.

[6] DIEULIN A., de MARSILY G. BEAUDOIN B. - Sur l'existence d'un effet de parcours dans le transfert d'éléments en solution en milieu poreux. CRAS, série $B, 1981$.

17] MATHERON G., de MARSILY G. - Is transport in porous media always diffusive? A counter example. $W R R$, vol. 16, $\mathrm{n}^{\circ}$ 5, Octobre 1980.

[8] GELHAR L.W., GUTJAHR A.L., NAFF R.L. - Stochastic analysis of macrodispersion in a stratified aquifer. WRR, vol. $15, n^{\circ} 6$, Décembre 1979.

[9] MARTIN J.M. - Déplacements miscibles dans des milieux poreux naturels de grande extension. Revue de l'IFP, XXVI, $n^{\circ} 11,1971$. 